\title{
Sleep Patterns with Obstructive Sleep Apnea in Middle Adolescence: A Cross-Sectional Study of Obese Patients
}

\author{
Olga Berdina, PhD; ; Irina Madaeva, $\mathrm{PhD}, \mathrm{ScD}$; Svetlana Bolshakova, $\mathrm{PhD}$; \\ Olga Bugun, PhD, ScD; Lyubov Rychkova, Corresponding Member of the RAS \\ Scientific Centre for Family Health and Human Reproduction Problems \\ Irkutsk, the Russian Federation
}

\begin{abstract}
Background: The aim of this study was to explore specific sleep patterns in obese adolescents aged 15-17 using polysomnography (PSG), whether or not obstructive sleep apnea (OSA) is present.

Methods and Results: Anthropometric measurements were taken of all participants, and outpatient PSGs were performed. After PSG, all obese participants were divided into two groups: non-OSA group $(\mathrm{n}=18)$ and OSA group $(\mathrm{n}=27)$. Fifteen lean adolescents were included in the control group. Apnea/hypopnea index, episodes of snoring, time with $\mathrm{SpO}_{2}<90 \%$, arousal index, sleep stages 1-2 were significantly higher; and lowest $\mathrm{SpO}_{2}$, sleep efficiency, slow wave sleep, rapid eye movement sleep were significantly lower in OSA patients than in both non-OSA groups. However, all PSG variables in the non-OSA obese group were comparable to lean controls.

Conclusion: Sleep characteristics are related to the presence of OSA, but not to the excess weight. Future research should evaluate sleep pattern changes following successful treatment of OSA in obese adolescents and longitudinal outcomes in adults. (International Journal of Biomedicine. 2020;10(3):235-240.)
\end{abstract}

Key Words: obstructive sleep apnea $\bullet$ obesity $\bullet$ polysomnography $\bullet$ middle adolescence

\section{Abbreviations}

AI, arousal index; AHI, apnea/hypopnea index; BMI, body mass index; CPAP, continuous positive airway pressure; EEG, encephalography; OSA, obstructive sleep apnea; PSG, polysomnography; REM, rapid eye movement; $\mathbf{S p O}_{2}$, arterial oxygen saturation; SE, sleep efficiency; SWS, slow wave sleep; zBMI, body mass index-for-age z-score.

\section{Introduction}

Obesity has become an epidemic in industrialized and developing countries among adults and children. About $10 \%$ of the world's school-aged children are estimated to be carrying excess body fat with an increased risk of developing chronic diseases, such as type 2 diabetes, heart disease and a variety of other comorbidities before or during early adulthood. ${ }^{(1,2)}$ It is known that many factors promote pediatric obesity (e.g.

*Corresponding author: Olga Berdina, PhD, MD. Leading researcher, Laboratory of Somnology and Neurophysiology, Scientific Centre for Family Health and Human Reproduction Problems. Irkutsk, Russia.E-mail: goodnight_84@mail.ru low physical activity, high calorie or high fat food, stress), but changing established weight-related habits is very difficult, and interventions targeting these behaviors have shown only moderate success. ${ }^{(3-5)}$ Thus, research is needed to elucidate additional mechanisms of excessive weight gain in children and adolescents to further improve obesity prevention and intervention efforts. Sleep has been proposed as a promising target. Meta-analyses indicate that not meeting recommended sleep hygiene in adolescence increases risk of developing overweight/obesity. ${ }^{(6)} \mathrm{C}$. Hart et al. ${ }^{(7)}$ published a hallmark review of pediatric sleep and obesity, which indicated that sleep duration, quality, and schedules were all related to BMI in youth. Hypotheses that sleep is linked to the development and maintenance of obesity propose that it impacts weight 
through a variety of biological and behavioral pathways. ${ }^{(8)}$ It has been noted that poor sleep quality may impact overall sleep duration and additionally may interfere with the natural cycles of sleep waves throughout the night, which can alter metabolism. ${ }^{(9)}$ Conversely, excessive weight is a risk factor for sleep-disordered breathing, including OSA and the likelihood of developing OSA in obese children is four to five times greater than in a healthy-weight peer. ${ }^{(10,11)}$ It is known that OSA is characterized by the collapse of the upper airway during sleep, leading to airflow cessation or reduction, intermittent hypoxemia, hypercapnia and sleep fragmentation. ${ }^{(12)}$ Untreated OSA may lead to an increased incidence of cardiovascular diseases, strokes, diabetes, depression, cognitive impairment and others. ${ }^{(13,14)}$

Adolescence (especially the ages of 15-17 years, or middle adolescence) is a time of important physical, emotional, behavioral and social changes. ${ }^{(15)}$ Sleep is a primary aspect of adolescent development. It is known that during adolescence, physiological sleep patterns (e.g. reduced depth of REM sleep, shortened total sleep time) and psychosocial influences on sleep change. ${ }^{(16-18)}$ Thereby, on the one hand, adolescents may be particularly vulnerable to sleep-related changes in their weight. ${ }^{(19,20)}$ On the other hand, as the pediatric obesity epidemic continues, adolescents comprise one of the fastest growing risk groups for developing OSA. ${ }^{(21,22)}$ This raises the possibility of a causal relationship, and therefore the potential for sleep-related interventions. However, a few studies in population-based samples have shown an association between obesity and sleep variables (and/or sleep abnormalities, e.g. OSA) in adolescence, but reported findings are not similar. Moreover, a major weakness of the research in this area is the exclusive reliance on self-reported sleep measures or actigraphy as objective measures of sleep. PSG, as a comprehensive method for assessing sleep, during which various physiological parameters are recorded, is the gold standard for diagnosing sleep-disordered breathing, including OSA. It allows accurately detecting sleep disorders, in order to timely and adequately choose treatment approaches, preventing the progression of sleep disturbances and their possible complications. ${ }^{(23)}$

To address these weaknesses, we conducted the study to explore specific sleep patterns in obese adolescents aged 1517 using PSG, whether or not OSA is present.

\section{Materials and Methods}

The present cross-sectional study, in which all variables were measured at the same time, was designed to explorer specific sleep patterns in obese adolescents with and without OSA by outpatient PSG. Study participants (45 adolescents aged 15-17 years) were recruited from obese patients referred to the Children's Clinic of Scientific Centre for Family Health and Human Reproduction Problems (Irkutsk, Russian Federation), in the period from October 2018 to May 2019. After PSG, all obese participants were divided into two groups: non-OSA group $(n=18)$ and OSA group $(n=27)$. Fifteen lean adolescents were included in the control group.

Study inclusion criteria: the 15-17 year age range; obesity, if zBMI $>2$ for age and sex, normal weight, if zBMI $\geq-2$ to +1 for age and sex; signed informed consent form.

Study non-inclusion critera: unwillingness to participate in this study, overweight or underweight (zBMI $>+1$ but $\leq+2$ and $\leq-2$ for age and sex, accordingly), craniofacial anomalies, secondary obesity, CPAP therapy.

Anthropometric measurements were taken of all participants, and outpatient PSGs were performed. Anthropometric parameters of adolescents were assessed once when they were included in the study. Height was measured to the nearest $0.1 \mathrm{~cm}$ using a stadiometer and weight was measured to the nearest $0.1 \mathrm{~kg}$. The adolescents wore light indoor clothing and no shoes during the measurements. BMI was calculated as $\mathrm{kg} / \mathrm{m}^{2}$. BMI was further analyzed as zBMI using the AnthroPlus calculator based on the WHO references. ${ }^{(24)}$

Due to the purpose of this study, the sleep patterns were evaluated objectively, using outpatient PSG, which was performed at the adolescent's usual sleep times. A technologist from the Sleep Center went to the Children's Clinic to place the leads. Adolescents were asked to record lights off and lights on times. They were supplied with an emergency telephone number to call during the night with questions, as well as a diagram and simple instruction sheet on replacing leads if they happened to notice that any fell off during the night. PSG data were collected using the AURA PSG GRASSTECHNOLOGIES (USA). We recorded six EEG derivations (Fp3-A2, Fp4-A1, C3-A2, C4-A1, O1-A2, and O2-A1), left and right electrooculography channels, submental muscle tonus, heart rate by electrocardiogram, airflow using a threepronged thermistor (Pro-Tech Services, Inc., Mukilteo, WA), nasal pressure by a pressure transducer (Pro-Tech Services, Inc., Walnut Cove, NC), snoring, chest and abdominal wall motions using respiratory inductance plethysmography, $\mathrm{SpO}_{2}$ by pulse oximetry, and body position. The recording was terminated by the adolescent in the morning after he/she awoke for the day, and the equipment was picked up by the technologist. Studies were transmitted electronically to the Sleep Center where they were scored by a single registered polysomnographic technologist and then interpreted by a single sleep medicine physician, using the American Academy of Sleep Medicine (AASM) ${ }^{(25)}$ scoring rules. Obstructive apneas were defined as at least a $90 \%$ reduction in the thermal signal amplitude, whereas hypopneas were defined as diminution of at least $30 \%$ of the nasal pressure signal that was associated with an arousal or desaturation of $3 \%$. Obstructive apneas and hypopneas were expressed as the number of apneas/hypopneas per hour of total sleep. OSA was identified if $\mathrm{AHI} \geq 2 \mathrm{n} / \mathrm{h} .{ }^{(26)}$ Cortical arousals were expressed as total number of arousals per $\mathrm{h}$ of total sleep time (AI). All studies were rated for technical quality. The percentage of total recording time with a satisfactory signal was visually assessed for each polysomnographic channel. A signal was considered satisfactory if the waveform was free of artifacts and the data were interpretable. Power EEG spectrum was automatically scored for consecutive 20-s epochs with Spectral Analysis option of GRASS-TELEFACTOR PSG Twin 4.5.2.

The study was conducted in accordance with ethical principles of the WMA Declaration of Helsinki (1964, ed. 2013) 
and approved by the Scientific Centre's for Family Health and Human Reproduction Problems Ethics Biomedical Committee of February 23, 2018 (Reference number 2018/2).

Statistical analysis was performed using the Statistica v10.0 software package (Stat-Soft Inc., USA). Baseline characteristics were summarized as frequencies and percentages for categorical variables and as mean \pm standard deviation (SD) for continuous variables. Multiple comparisons were performed with one-way ANOVA and Tukey's HSD Post-hoc Test. Group comparisons with respect to categorical variables were performed using chi-square tests or, alternatively, Fisher's exact test. A probability value of $P<0.05$ was considered statistically significant.

\section{Results}

Participants' characteristics were analyzed (Table 1). Adolescents with obesity $(n=45)$ had a mean age of 16.3 year, whereas lean adolescents $(\mathrm{n}=15)$ had mean age of 16.0 years $(P=0.56)$. However, zBMI for lean controls differed significantly from that for obese groups $(P<0.0001)$. Females comprised $46.7 \%$ and $44.5 \%$ of corresponding samples $(P=0.88)$. OSA was present in $60 \%$ of patients with obesity, wherein there were 2 times more boys than girls. It should be noted that OSA has a sexual dimorphism, so men are especially vulnerable to sleep apnea, compared to women. ${ }^{(27,28)}$

Table 1.

Comparison for age, gender, BMI and presence of OSA between the entire obese sample and the lean group

\begin{tabular}{|l|c|c|c|}
\hline \multicolumn{1}{|c|}{ Variables } & Obese group $(\mathrm{n}=45)$ & Lean group $(\mathrm{n}=15)$ & $P$-value \\
\hline Age, y & $16.3 \pm 0.2$ & $16.0 \pm 0.5$ & 0.56 \\
\hline Female, n (\%) & $20(44.5)$ & $7(46.7)$ & 0.88 \\
\hline BMI, kg/m² & $33.6 \pm 3.1$ & $19.0 \pm 2.4$ & $<0.0001$ \\
\hline zBMI & $2.4 \pm 0.9$ & $-0.08 \pm 0.4$ & $<0.0001$ \\
\hline $\begin{array}{l}\text { OSA, n (\%) } \\
\text { Male, n (\%) }\end{array}$ & $\begin{array}{c}27(60) \\
19(70.4)\end{array}$ & - & $<0.0001$ \\
\hline
\end{tabular}

Characteristics and PSG data of patients with and without OSA were separately analyzed (Table 2). The OSA group was similar to the rest of the non-OSA group in terms of age (16.3 \pm 0.5 year vs. $16.1 \pm 0.3$ year) and zBMI ( $2.5 \pm 0.5$ vs. $2.4 \pm 0.7$ ), but both obese groups had significantly higher BMI compared to lean controls $(P<0.0001)$. Polysomnography comparisons demonstrated significant differences between the obese subjects with OSA and both obese controls and lean controls in terms of severity of OSA, gas exchange during sleep, frequency of arousals, sleep efficiency and percentage of sleep stages. ANOVA showed that AHI, episodes of snoring, time with $\mathrm{SpO}_{2}<90 \%$, AI, sleep stages $1-2$ were significantly higher; and lowest $\mathrm{SpO}_{2}$, SE, SWS, REM sleep were significantly lower in OSA patients than in both nonOSA groups. However, all PSG variables in the non-OSA obese group were comparable to lean controls.

Generally, nocturnal sleep structure changes in OSA obese adolescents were characterized by an increase in the duration and a reduction in number of sleep cycles, and segmentation of non-REM sleep and REM sleep, compared with both non-OSA groups. Furthermore, increased frequency of arousals are accompanied by frequent sharp transients and periods of hypersynchronous theta activity of the EEG. The dominant frequency of EEG oscillations in nocturnal sleep in OSA obese adolescents was $6.8 \pm 0.73 \mathrm{~Hz}$, which is significantly lower than the similar variables in both control groups $(10.7 \pm 0.79 \mathrm{~Hz}$ and $11.3 \pm 0.32 \mathrm{~Hz}$, accordingly, $P<0.01)$. One of the highlights was the significant decrease in the sleep efficiency in patients with obesity and OSA.

Table 2.

Participants' and PSG characteristics in obese adolescents according to the presence or absence of OSA and in lean controls

\begin{tabular}{|l|c|c|c|c|}
\hline \multicolumn{1}{|c|}{ Variables } & $\begin{array}{c}\text { Obese } \\
\text { adolescents } \\
\text { with OSA } \\
(\mathrm{n}=27)\end{array}$ & $\begin{array}{c}\text { Obese } \\
\text { adolescents } \\
\text { without OSA } \\
(\mathrm{n}=18)\end{array}$ & $\begin{array}{c}\text { Lean } \\
\text { controls } \\
(\mathrm{n}=15)\end{array}$ & $P$-value \\
\hline Age, y & $16.3 \pm 0.5$ & $16.1 \pm 0.3$ & $16.0 \pm 0.5$ & 0.67 \\
\hline BMI, kg/m & $34.1 \pm 2.4$ & $32.0 \pm 2.5$ & $19.0 \pm 2.4$ & $<0.0001^{1}$ \\
\hline zBMI & $2.5 \pm 0.5$ & $2.4 \pm 0.7$ & $-0.08 \pm 0.4$ & $<0.0001^{1}$ \\
\hline AHI, n/h & $13.2 \pm 1.8$ & $1.1 \pm 0.3$ & $1.0 \pm 0.1$ & $<0.0001^{2}$ \\
\hline Snore, n & $1087.8 \pm 557.5$ & $267.4 \pm 31.6$ & $243.6 \pm 26.7$ & $<0.0001^{2}$ \\
\hline Baseline SpO ${ }_{2}^{2} \%$ & $97.8 \pm 1.6$ & $98.2 \pm 0.4$ & $98.6 \pm 0.4$ & 0.84 \\
\hline Lowest SpO ${ }_{2} \%$ & $85.3 \pm 1.6$ & $95.6 \pm 0.2$ & $96.1 \pm 0.6$ & $<0.0005^{2}$ \\
\hline Time with SpO 2 \\
$<90 \%, \%$ TST
\end{tabular}

${ }^{1}$ comparison between lean controls and both obese groups;

${ }^{2}$ comparisons between OSA and both control groups (the non-OSA obese group and lean controls). 
Sleep organization analysis in obese adolescents with and without OSA revealed the following: a change in superficial sleep with its minimum value in the first cycles and an increase in duration in the second, third and fourth cycles; a decrease in duration of deep sleep and atypical delta sleep, and a significant decrease in REM duration, predominantly in the second, third and fourth cycles in OSA obese patients.

\section{Discussion}

In the present study, we found sleep disturbance such as OSA in more than half of obese adolescents referred for PSG. This finding is consistent with recent literature. Andersen et al. ${ }^{(29)}$ demonstrated the presence of OSA in $44.6 \%$ of the overweight study population and showed that a one-unit increase in the zBMI equaled an average increase in the AHI of $35 \%$. A lower OSA frequency may be due to the inclusion in this study of children and adolescents with zBMI $>1.28$ (BMI $>90$ th percentile according to age- and sex-adjusted reference) compared to $\mathrm{zBMI}>2$ (BMI $>95$ th percentile according to age- and sex-adjusted reference). Kang et al., ${ }^{(30)}$ Evangelisti et al., ${ }^{(31)}$ Lennon et al., ${ }^{(32)}$ and other scientists have shown that overweight status is negatively correlated with minimal $\mathrm{SpO}_{2}$ but positively correlated with $\mathrm{AHI}$, and that obese children and adolescents have a significantly higher risk of OSA than children with underweight and normal weight status. The association of OSA and obesity was also reported by Parmar et al., ${ }^{(33)}$ Scheffler et al. ${ }^{(34)}$ and others who demonstrated that using CPAP and adenotonsillectomy are effective treatments for OSA in obese subjects, and can improve OSA-related parameters such as $\mathrm{AHI}$ and $\mathrm{SpO}_{2}$ as well as some of the cardiometabolic dysfunctions linked to the disorder. ${ }^{(35)}$ In contrast, Andersen et al. ${ }^{(36)}$ reported that OSA improved during obesity treatment (e.g. lifestyle changes, physical activities, diet, behavioral therapy and so on) and the reduction in BMI was significantly associated with the reduction in AHI.

It is known that the integrative processes that occur in the brain during sleep determine the sequence of occurrence of different phases and stages (sleep homeostasis) and characterize the ability of brain systems to achieve the main purposes of sleep - restoration and saving of metabolic health. ${ }^{(37)}$ Any adverse effect on the body, primarily on the brain, can inevitably lead to sleep disruption of homeostasis and its restorative capabilities. ${ }^{(38)}$ As expected, under the influence of periodic nocturnal hypoxia, the structural organization of sleep also changed in our patients with OSA.

It is argued that sleep duration and sleep quality have been implicated as risk factors for obesity. Poor sleep quality may impact overall sleep duration and additionally may interfere with the natural cycles of sleep waves throughout the night, which can alter metabolism. ${ }^{(9)}$ Obese adolescents had more disrupted sleep and less sleep efficiency than the healthy-weight controls. ${ }^{(21,39)}$ It is interesting to note that we did not find changes in total sleep duration in adolescents with obesity, compared to lean controls, as well as between OSA and non-OSA obese patients. However, sleep efficiency and sleep structure were significantly changed in obese adolescents with OSA only, but not in obese controls, which were comparable to the lean group. These findings are different from the results demonstrated by Xanthopoulos et al., ${ }^{(22)}$ who did not find differences in sleep efficiency and duration of sleep stages between OSA obese patients and obese controls, but reported significantly higher sleep arousals in OSA participants, compared to both the non-OSA obese group and lean controls, which was also shown in our study.

In conclusion, this study demonstrates that more than half of an obese sample was affected by OSA. Further, obese adolescents with OSA showed altered sleep patterns, as compared to the non-OSA obese patients and lean controls. We can assume that sleep characteristics are related to the presence of OSA, but not to the excess weight. So excessive respiratory stimuli in OSA can break sleep homeostasis due to an increase in the ability of the sleeping brain to provoke arousal as a putative mechanism to protect from arousing stimuli, and thereby enhancing sleep consolidation. Our findings assume that untreated OSA during adolescence may lead to obesity progression and development of complications (e.g. neurobehavioral deficits, cardiometabolic changes, etc.) in adulthood. Physicians should identify adolescents from OSA risk groups, and arrange timely and adequate therapy to prevent significant sleep homeostasis changes, as well as OSA-linked disorders. It is assumed that a chronic care, multidisciplinary obesity treatment, as well as CPAP therapy, should be considered among the first-line treatments of OSA (mostly severe) in management of adolescents. Future research should evaluate sleep pattern changes following successful treatment of OSA in obese adolescents and longitudinal outcomes in adults.

\section{Competing Interests}

The authors declare that they have no competing interests.

\section{References}

1. Daniels SR. Complications of obesity in children and adolescents. Int J Obes (Lond). 2009;33 Suppl 1:S60-S65. doi:10.1038/ijo.2009.20

2. Arroyo-Johnson C, Mincey KD. Obesity Epidemiology Worldwide. Gastroenterol Clin North Am. 2016;45(4):571579. doi:10.1016/j.gtc.2016.07.012

3. Darenskaya MA, Rychkova LV, Kolesnikov SI, Gavrilova OA, Kravtsova OV, Grebenkina LA, et al. Oxidative stress parameters in adolescent boys with exogenous-constitutional obesity. Free Radic. Biol. Med. 2017;112:129-130. doi: 10.1016/j.freeradbiomed.2017.10.195.

4. Hagman E, Danielsson P, Brandt L, Svensson V, Ekbom A, Marcus C. Childhood Obesity, Obesity Treatment Outcome, and Achieved Education: A Prospective Cohort Study. J Adolesc Health. 2017;61(4):508-513. doi:10.1016/j. jadohealth.2017.04.009

5. Pogodina A, Rychkova L, Kravtzova O, Klimkina J, Kosovtzeva A. Cardiometabolic Risk Factors and Health- 
Related Quality of Life in Adolescents with Obesity. Child Obes. 2017;13(6):499-506. doi:10.1089/chi.2016.0330

6. Chen X, Beydoun MA, Wang Y. Is sleep duration associated with childhood obesity? A systematic review and meta-analysis. Obesity (Silver Spring). 2008;16(2):265-274. doi:10.1038/oby.2007.63

7. Hart CN, Cairns A, Jelalian E. Sleep and obesity in children and adolescents. Pediatr Clin North Am. 2011;58(3):715-733. doi:10.1016/j.pcl.2011.03.007

8. Kreitsch KN, Chardon ML, Beebe DW, Janicke DM. Sleep and weight-related factors in youth: A systematic review of recent studies. Sleep Med Rev. 2019;46:87-96. doi:10.1016/j. smrv.2019.04.010

9. McHill AW, Wright KP Jr. Role of sleep and circadian disruption on energy expenditure and in metabolic predisposition to human obesity and metabolic disease. Obes Rev. 2017;18 Suppl 1:15-24. doi:10.1111/obr.12503

10. Qu XX, Esangbedo IC, Zhang XJ, Liu SJ, Li LX, Gao $\mathrm{S}$, et al. Obstructive Sleep Apnea Syndrome is Associated with Metabolic Syndrome among Adolescents and Youth in Beijing: data from Beijing Child and Adolescent Metabolic Syndrome Study [published correction appears in Chin Med J (Engl). 2015 Dec 20;128(24):3390]. Chin Med J (Engl). 2015;128(17):2278-2283 . doi:10.4103/03666999.163394

11. Su M-S, Zhang H-L, Cai X-H, Lin Y, Liu PN, Zhang $\mathrm{YB}$, et al. Obesity in children with different risk factors for obstructive sleep apnea: a community-based study. Eur J Pediatr. 2016;175(2):211-220. doi:10.1007/s00431-0152613-6

12. Marcus CL, Brooks IJ, Ward SD, Gozal D, Halbower AC, Jones J, et al. Diagnosis and management of childhood obstructive sleep apnea syndrome. Pediatrics. 2012;130(3):e714-e755. doi:10.1542/peds.2012-1672

13. Morsy NE, Farrag NS, Zaki NFW, Badawy AY, Abdelhafez SA, El-Gilany A-H, et al. Obstructive sleep apnea: personal, societal, public health, and legal implications. Rev Environ Health. 2019;34(2):153-169. doi:10.1515/reveh-2018-0068

14. Jackson ML, Tolson J, Bartlett D, Berlowitz DJ, Varma $\mathrm{P}$, Barnes M. Clinical depression in untreated obstructive sleep apnea: examining predictors and a meta-analysis of prevalence rates. Sleep Med. 2019;62:22-28. doi:10.1016/j. sleep.2019.03.011

15. Gaete V. [Adolescent psychosocial development]. Rev Chil Pediatr. 2015;86(6):436-443. doi:10.1016/j.rchipe.2015.07.005. [Article in Spanish].

16. Wolfson AR, Carskadon MA. Sleep schedules and daytime functioning in adolescents. Child Dev. 1998;69(4):875-887.

17. Hagenauer MH, Lee TM. Adolescent sleep patterns in humans and laboratory animals. Horm Behav. 2013;64(2):270279. doi:10.1016/j.yhbeh.2013.01.013

18. Berdina O, Madaeva I, Bolshakova S, Tsykunova M, Bugun O, Rychkova L. Applying a Translated Version of the Adolescent Sleep Habits Survey in Russian High School Children with Obesity. International Journal of Biomedicine. 2020;10(1):61-65. doi: 10.21103/Article10(1)_OA10.

19. Beebe DW, Lewin D, Zeller M, McCabe M, MacLeod K, Daniels SR, et al. Sleep in overweight adolescents: shorter sleep, poorer sleep quality, sleepiness, and sleep-disordered breathing. J Pediatr Psychol. 2007;32(1):69-79. doi:10.1093/ jpepsy/jsj104

20. Hayes JF, Balantekin KN, Altman M, Wilfley DE, Taylor
CB, Williams J. Sleep Patterns and Quality Are Associated with Severity of Obesity and Weight-Related Behaviors in Adolescents with Overweight and Obesity. Child Obes. 2018;14(1):11-17. doi:10.1089/chi.2017.0148

21. Verhulst SL, Van Gaal L, De Backer W, Desager $\mathrm{K}$. The prevalence, anatomical correlates and treatment of sleep-disordered breathing in obese children and adolescents. Sleep Med Rev. 2008;12(5):339-346. doi:10.1016/j.smrv.2007.11.002

22. Xanthopoulos MS, Gallagher PR, Berkowitz RI, Radcliffe J, Bradford R, Marcus CL. Neurobehavioral functioning in adolescents with and without obesity and obstructive sleep apnea. Sleep. 2015;38(3):401-410. Published 2015 Mar 1. doi: $10.5665 /$ sleep. 4498

23. Rundo JV, Downey R 3rd. Polysomnography. Handb Clin Neurol. 2019;160:381-392. doi:10.1016/B978-0-444-64032$1.00025-4$

24. World Health Organization. Growth reference 5-19 years. BMI-for-age (5-19 years). [Electronic resource]. https://www. who.int/growthref/who2007_bmi_for_age/en/.

25. Berry RB, Brooks R, Gamaldo $\overline{\mathrm{C}} \mathrm{E}$, Harding SM, Lloyd RM, Marcus CL, et al. The AASM Manual for the scoring of sleep and associated events: rules, terminology and technical specifications. Version 2.2. Darien, Illinois: American Academy of Sleep Medicine; 2015.

26. Kaditis AG, Alvarez MLA, Boudewyns A, Alexopoulos EI, Ersu R, Joosten K, et al. Obstructive sleep disordered breathing in 2- to 18-year-old children: diagnosis and management. Eur Respir J. 2016;47(1):69-94. doi:10.1183/13993003.003852015

27. Senaratna CV, English DR, Currier D, Perret JL, Lowe A, Lodge C, et al. Sleep apnoea in Australian men: disease burden, co-morbidities, and correlates from the Australian longitudinal study on male health. BMC Public Health. 2016;16(Suppl 3):1029. Published 2016 Oct 31. doi:10.1186/ s12889-016-3703-8

28. Madaeva I, Berdina O, Kolesnikova L, Semenova N. Gender-related differences of sleep pattern characteristics in obstructive sleep apnea. Sleep Med. 2017;40(Suppl 1):e204-5. doi: 10.1016/j.sleep.2017.11.598.

29. Andersen IG, Holm, Homøe P. Obstructive sleep apnea in children and adolescents with and without obesity. Eur Arch Otorhinolaryngol. 2019;276(3):871-878. doi:10.1007/ s00405-019-05290-2

30. Kang KT, Lee PL, Weng WC, Hsu WC. Body weight status and obstructive sleep apnea in children. Int J Obes (Lond). 2012;36(7):920-924. doi:10.1038/ijo.2012.5

31. Evangelisti M, Shafiek H, Rabasco J, Forlani M, Montesano M, Barreto M, et al. Oximetry in obese children with sleep-disordered breathing. Sleep Med. 2016;27-28:8691. doi:10.1016/j.sleep.2016.08.014

32. Lennon CJ, Wang RY, Wallace A, Chinnadurai S. Risk of failure of adenotonsillectomy for obstructive sleep apnea in obese pediatric patients. Int $\mathrm{J}$ Pediatr Otorhinolaryngol. 2017;92:7-10. doi:10.1016/j.ijporl.2016.09.026

33. Parmar A, Baker A, Narang I. Positive airway pressure in pediatric obstructive sleep apnea. Paediatr Respir Rev. 2019;31:43-51. doi:10.1016/j.prrv.2019.04.006

34. Scheffler P, Wolter NE, Narang I, Amin R, Holler T, Ishman SL, et al. Surgery for Obstructive Sleep Apnea in Obese Children: Literature Review and Metaanalysis. Otolaryngol Head Neck Surg. 2019;160(6):985-992. doi:10.1177/0194599819829415 
35. Patinkin ZW, Feinn R, Santos M. Metabolic Consequences of Obstructive Sleep Apnea in Adolescents with Obesity: A Systematic Literature Review and Meta-Analysis. Child Obes. 2017;13(2):102-110. doi:10.1089/chi.2016.0248

36. Andersen IG, Holm JC, Homøe P. Impact of weightloss management on children and adolescents with obesity and obstructive sleep apnea. Int J Pediatr Otorhinolaryngol. 2019;123:57-62. doi:10.1016/j.ijporl.2019.04.031

37. Donlea JM. Neuronal and molecular mechanisms of sleep homeostasis. Curr Opin Insect Sci. 2017;24:51-57. doi:10.1016/j.cois.2017.09.008

38. Olini N, Rothfuchs I, Azzinnari D, Pryce CR, Kurth $\mathrm{S}$, Huber R. Chronic social stress leads to altered sleep homeostasis in mice. Behav Brain Res. 2017;327:167-173. doi:10.1016/j.bbr.2017.03.022

39. Miller AL, Lumeng JC, LeBourgeois MK. Sleep patterns and obesity in childhood. Curr Opin Endocrinol Diabetes Obes. 2015;22(1):41-47. doi:10.1097/MED.0000000000000125 